\title{
Preparation of Cefquinome Nanoparticles by Using the Supercritical Antisolvent Process
}

\author{
Xiao Kefeng, ${ }^{1,2,3}$ Wang Weiqiang, ${ }^{1,3}$ Hu Dedong, ${ }^{3,4}$ Hao Zhihui, \\ Qu Yanpeng, ${ }^{1,3}$ and Liu Yan ${ }^{1,3}$ \\ ${ }^{1}$ School of Mechanical Engineering, Shandong University, Jinan 250061, China \\ ${ }^{2}$ College of Chemistry and Pharmaceutical Science, Qingdao Agricultural University, Qingdao 266109, China \\ ${ }^{3}$ Shanda-Lunan Research Institute of Supercritical Fluid Technology, Shandong University, Jinan 250061, China \\ ${ }^{4}$ The College of Electromechanical Engineering, Qingdao University of Science and Technology, Qingdao 266061, China
}

Correspondence should be addressed to Wang Weiqiang; wqwang@sdu.edu.cn

Received 15 September 2015; Accepted 5 November 2015

Academic Editor: John Zhanhu Guo

Copyright (C) 2015 Xiao Kefeng et al. This is an open access article distributed under the Creative Commons Attribution License, which permits unrestricted use, distribution, and reproduction in any medium, provided the original work is properly cited.

\begin{abstract}
The supercritical antisolvent process was used successfully to prepare nanoparticles of cefquinome. These particles were observed by scanning electron microscope (SEM) and their average diameter was measured by laser particle size analyzer. In the experiments, dimethyl sulfoxide (DMSO) was selected as solvent to dissolve cefquinome sulfate. It was confirmed by orthogonal experiments that the concentration of solution was the primary factor in this process followed by feeding speed of solution, precipitation pressure, and precipitation temperature. Moreover, the optimal conditions of preparing nanoparticles of cefquinome by supercritical antisolvent process were that solution concentration was $100 \mathrm{mg} / \mathrm{mL}$, solution flow speed was $1.5 \mathrm{~mL} / \mathrm{min}$, operating pressure was $13 \mathrm{Mpa}$, and operating temperature was $33^{\circ} \mathrm{C}$. Confirmatory experiment was conducted under this condition. It was found that the appearance of particles was flakes and the average diameter of particles was 0.71 microns. Finally, influence law of individual factor on particle size was investigated by univariate analysis.
\end{abstract}

\section{Introduction}

Cefquinome, one of the fourth-generation animal dedicated cephalosporin antibiotics, was approved by the committee for medicinal products for veterinary use (CVMP) for respiratory tract infections, cows udder inflammation, respiratory disease of pigs, horses sepsis, and other serious infections caused by susceptible strains in swine CP and cattle [1,2]. Cefquinome has many advantages, such as strong antibacterial activity, good pharmacokinetic characteristics, little toxicity, and low residual [3]. Compared with the secondand third-generation cephalosporins, cefquinome has better broad-spectrum antimicrobial activity, higher bioavailability, and more broad application prospects. However, the size of existing cefquinome particle is greater than 2 microns. The large particle size of cefquinome leads to slow absorption rate and low bioavailability by either topical or injectable applications [4]. According to Ostwald-Freundlich equation, when the particle size of drug is less than 1 micron, the solubility of the drug would increase greatly [5], thereby increasing the rate of absorption and bioavailability. Therefore, it is urgent to study and prepare nanoscaled cefquinome particles. At present, the preparation technologies of ultrafine particles in pharmaceutical field include spray drying, emulsification [6], the antisolvent method [7], and supercritical fluid (SCF) method $[8,9]$. Because of the advantages of low operating temperature, no solvent residue, and controllable particle size, supercritical fluid method attracted more and more attention of researchers worldwide. The processes of preparing nanodrug by supercritical fluid include two categories: rapid expansion of supercritical solution (RESS) and supercritical antisolvent (SAS) [10-12]. Because most drugs cannot be dissolved in the supercritical fluid, RESS process is restricted and SAS process has applied more and more in nanodrug preparations. SAS precipitation is an alternative to the liquid antisolvent precipitation and can effectively 


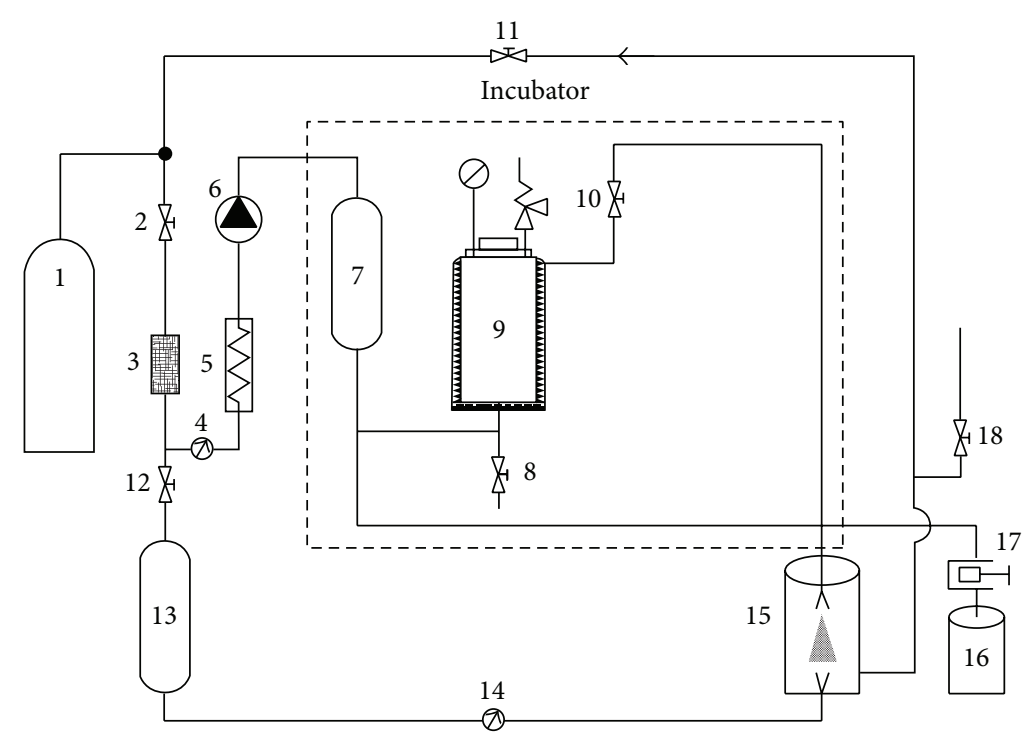

FIGURE 1: The flowchart of preparing nanoparticles by supercritical fluid process. 1: gas cylinder; 2: valve; 3: purifier; 4: flowmeter; 5: refrigeration equipment; 6: high-pressure pump; 7: buffer tank; 8: valve; 9: high-pressure mixing vessel; 10: valve; 11: back pressure valve; 12: back pressure valve; 13: separation tank; 14: flowmeter; 15: crystallizer; 16: solution tank; 17: metering pump; 18: drain valve.

overcome the limitations of liquid antisolvent processing, with the complete elimination of the solvents [13]. Moreover, it is easier to control the particle size of cefquinome in SAS process, and the operating temperature is low, a little higher than room temperature. Many researchers have focused on the application of SAS on nanodrug productions $[10,14]$. However, very few reported nanoscaled cefquinome with SAS process. In this study, we investigated the optimal conditions of preparing nanoparticles of cefquinome by SAS process and the influence law of individual factor on particle size by univariate analysis.

\section{Materials and Methods}

2.1. Materials. Cefquinome, with mass fraction purity of $82 \%$ (calculated by cefquinome content), was purchased from Qilu Shenghua Pharmaceutical Co., Ltd. Carbon dioxide, with purity of $99 \%$, was purchased from Jinan German Foreign Specialty Gases Co., Ltd. Dimethyl sulfoxide, with purity of analytical grade, was purchased from Tianjin Fuyu Fine Chemical Co., Ltd.

2.2. Equipment and Operating Procedure. The flowchart of preparing nanoparticles by supercritical fluid process was shown in Figure 1. In the process, the carbon dioxide from gas cylinder 1 was purified by purifier 3 and then was pressurized by high-pressure pump 6 to supercritical pressure slightly higher than the pressure required for the experiment after being cooled to $0-5^{\circ} \mathrm{C}$ liquid by cooling device 5 . The processed carbon dioxide was added into high-pressure mixing vessel 9 through buffer tank 7. Both high-pressure mixing vessel 9 and buffer tank 7 were placed in an incubator. The temperature of carbon dioxide can be controlled to the temperature required for the experiment by adjusting the temperature of the incubator. After that, valve 10 was opened and supercritical carbon dioxide spray into crystallization vessel 15 through the nozzle. The pressure in crystallization vessel 15 was adjusted by adjusting valve 18 . When the pressure reached predetermined pressure, the pure DMSO was added into solution tank 16 and was pumped by metering pump 17 into the nozzle and then sprayed from the nozzle. After several minutes, when the pressure was steady, the flow of the pure DMSO was stopped. The cefquinome/DMSO solution with certain concentration was added into solution tank 16 and was pumped by metering pump 17 into the nozzle at a given flow rate and then sprayed from the nozzle. After spraying out from the nozzle, the solution at high speed mixed sufficiently with supercritical carbon dioxide at high speed. Because of the antisolvent effect of supercritical carbon dioxide, the solute in the solution, cefquinome, precipitated rapidly. The solvent in the solution, DMSO, was carried out by carbon dioxide from valve 18. Because there was the sintered plate in crystallizer exit, the precipitated cefquinome particles gathered at the bottom of the crystallizer. After all of the solution in solution tank 16 was pumped into the nozzle, supercritical carbon dioxide continued to be pumped into the nozzle for sixty minutes so as to carry all remaining DMSO out of the crystallizer. Finally, valve 18 was opened slowly in order to decrease the pressure in crystallizer till the pressure was zero. Then the crystallizer was opened and the cefquinome particles were collected.

An orthogonal $\mathrm{L}_{9}\left(3^{4}\right)$ test design was utilized to investigate the optimal operating condition of cefquinome micronization process. The level range of each factor was based on the results of preliminary experiments and related references [11, 15]. As shown in Table 1, this experiment was fulfilled with four factors and three levels, namely, cefquinome concentration (100, 200, and $300 \mathrm{mg} / \mathrm{mL})$, feeding speed $(0.5,1$, and $1.5 \mathrm{~mL} / \mathrm{min})$, precipitation pressure 
TABLE 1: Factors and levels of the orthogonal array design.

\begin{tabular}{lcccc}
\hline Factor & $\begin{array}{c}\text { Concentration of } \\
\text { solution }(\mathrm{mg} / \mathrm{mL})\end{array}$ & $\begin{array}{c}\text { Cefquinome solution } \\
\text { feeding speed }(\mathrm{mL} / \mathrm{min})\end{array}$ & $\begin{array}{c}\text { Precipitation } \\
\text { pressure }(\mathrm{Mpa})\end{array}$ & $\begin{array}{c}\text { Precipitation } \\
\text { temperature }\left({ }^{\circ} \mathrm{C}\right)\end{array}$ \\
\hline 1 & 100 & 0.5 & 10 & 33 \\
2 & 200 & 1 & 13 & 38 \\
3 & 300 & 1.5 & 16 & 43 \\
\hline
\end{tabular}

(10, 13, and $16 \mathrm{MPa})$, and precipitation temperature (33, 38 , and $43^{\circ} \mathrm{C}$ ). The mean particle size (MPS) of prepared cefquinome $(\mathrm{nm})$ acted as the dependent variable changing with each factor.

2.3. Surface Morphology Analysis. The morphology of original cefquinome particles and micronized (nanoscaled) cefquinome particles was examined by SEM. The SEM was NOVA NANOSEM 450 type (American FEI Corporation). Cefquinome particles were stuck on the test bench with double-sided conductive tape and were sprayed gold. Then these particles were put in the SEM and observed at different magnifications.

2.4. Particle Diameter Analysis. MPS and particle size distribution (PSD) of processed cefquinome were determined by laser particle size analyzer (BT-9300H). Before examination, a little amount of cefquinome particles was put into the beaker filled with approximately $80 \mathrm{~mL}$ ultrapure water. Then the suspension was stirred and dispersed in an ultrasonic disperser for 5 minutes. At the same time, background test was conducted in a clean sample cell filled with ultrapure water. The suspension $4 \sim 6 \mathrm{~mL}$ was drawn by a special syringe from the central part of the suspension and was injected into the sample cell. Finally, test was conducted and the MPS of cefquinome particles were obtained.

\section{Results and Discussion}

3.1. Effect of Operating Conditions on Average Diameter. The particle size was influenced by two possible mechanisms, evaporation of the solvent into the antisolvent phase and diffusion of the antisolvent into the droplets [16]. Concentration of solution, solution feeding speed, and density of supercritical $\mathrm{CO}_{2}$ played an important role in mass transfer between organic solvents and $\mathrm{CO}_{2}$ during particle formation. The density of supercritical $\mathrm{CO}_{2}$ was dominated by pressure and temperature [14]. In SAS process, using the characteristics of higher solubility of organic solvents in SCF, the cefquinome/DMSO solution would expand suddenly when SCF as antisolvent was mixed with the solution. After expanding, the density of the solution became lighter, so that the solubility of cefquinome in DMSO decreased sharply. And almost at the same time the cefquinome became supersaturated in the solution and formed crystalline or amorphous particles [17]. The density of SCF and the saturation of the solution affect particle morphology, particle size, and particle size distribution greatly. The density of SCF was affected by pressure and temperature, and the saturation of the solution was influenced by the feeding speed of the solution and the concentration of the solution [18]. Therefore, concentration of solution, feeding speed, precipitation pressure, and precipitation temperature were chosen as the factors to study the process of preparing cefquinome nanoparticles by using SAS process. An orthogonal $\mathrm{L}_{9}\left(3^{4}\right)$ test design was used in the experiment and the experiment results were analyzed by using Design Expert 8.0 software according to statistical methods. The average diameter was selected as evaluation indicator. The analyzing results were shown in Table 2.

The bigger the range is, the more important the factor is. As shown in Table 2, the range of different factors was sorted in descending order as follows: $\mathrm{A}>\mathrm{B}>\mathrm{C}>\mathrm{D}$. Consequently, the effect of concentration of solution on the process was the biggest, followed by the feeding speed of cefquinome solution, precipitation pressure, and precipitation temperature. The smallest particle average diameter obtained in this series of experiments was $0.72 \mu \mathrm{m}$. But, according to test analyzing, this operating condition was not the best and the optimal operating condition should be $\mathrm{A}_{1} \mathrm{~B}_{3} \mathrm{C}_{2} \mathrm{D}_{1}$ that the concentration of solution was $100 \mathrm{mg} / \mathrm{mL}$; the feeding speed was $1.5 \mathrm{~mL} / \mathrm{min}$; precipitation pressure was $13 \mathrm{Mpa}$ and precipitation temperature was $33^{\circ} \mathrm{C}$. Confirmatory test was conducted in the optimal operating condition and the average diameter of prepared cefquinome particle was $0.71 \mu \mathrm{m}$.

3.2. Effect of Each Factor on the Process. The relationship of each factor and the average diameter of cefquinome particles were shown in Figure 2. The average diameter increased with the increase of concentration of the cefquinome. This factor had an important effect on the micronization of cefquinome. That is mainly because of the fact that, in SAS process, when precipitation occurred, crystal nuclei produced in low concentration solution were less than that in high concentration solution. Therefore, the crystal nuclei produced in low concentration solution grew slower than that in high concentration solution so that the average diameter of nanoparticles produced in low concentration solution was relatively small. Consequently, in the view of producing nanoparticle, the concentration of solution should be as low as possible. If the amount of drug treatment was taken into account, the concentration cannot be too low.

It can be concluded from Figure 2 that the average diameter decreased with increase of feeding speed. When the feeding speed increased, the solution can be mixed with supercritical carbon dioxide more sufficiently. And the solution expanded more rapidly so that the speed of cefquinome particle precipitation became larger. Consequently, the growing time of crystal nuclei became shorter and the produced 
TABLE 2: Analysis of $\mathrm{L}_{9}\left(3^{4}\right)$ test results.

\begin{tabular}{|c|c|c|c|c|c|}
\hline Run & $\begin{array}{l}\text { (A) Concentration } \\
\text { of solution }(\mathrm{mg} / \mathrm{mL})\end{array}$ & $\begin{array}{l}\text { (B) Cefquinome solution } \\
\text { feeding speed }(\mathrm{mL} / \mathrm{min})\end{array}$ & $\begin{array}{l}\text { (C) Precipitation } \\
\text { pressure (Mpa) }\end{array}$ & $\begin{array}{l}\text { (D) Precipitation } \\
\text { temperature }\left({ }^{\circ} \mathrm{C}\right)\end{array}$ & $\begin{array}{c}\text { Average } \\
\text { diameter }(\mu \mathrm{m})\end{array}$ \\
\hline 1 & 1 & 1 & 1 & 1 & 0.92 \\
\hline 2 & 1 & 2 & 2 & 2 & 0.72 \\
\hline 3 & 1 & 3 & 3 & 3 & 0.88 \\
\hline 4 & 2 & 1 & 2 & 3 & 1.11 \\
\hline 5 & 2 & 2 & 3 & 1 & 1.05 \\
\hline 6 & 2 & 3 & 1 & 2 & 0.91 \\
\hline 7 & 3 & 1 & 3 & 2 & 2.06 \\
\hline 8 & 3 & 2 & 1 & 3 & 1.12 \\
\hline 9 & 3 & 3 & 2 & 1 & 0.96 \\
\hline$K_{1}^{\mathrm{a}}$ & 0.84 & 1.363 & 0.983 & 0.977 & \\
\hline$K_{2}$ & 1.023 & 0.963 & 0.93 & 1.23 & \\
\hline$K_{3}$ & 1.38 & 0.917 & 1.33 & 1.037 & \\
\hline$R^{\mathrm{b}}$ & 0.54 & 0.446 & 0.4 & 0.253 & \\
\hline Optimal level & $\mathrm{A}_{1}$ & $\mathrm{~B}_{3}$ & $\mathrm{C}_{2}$ & $\mathrm{D}_{1}$ & \\
\hline
\end{tabular}

${ }^{\mathrm{a}} K_{i}{ }^{A}=\Sigma\left(\right.$ mean particle size at $\left.A_{i}\right) / 3$, the mean values of mean particle size for a certain factor at each level with standard deviation.

${ }^{\mathrm{b}} R_{i}{ }^{A}=\max \left\{K_{i}{ }^{A}\right\}-\min \left\{K_{i}{ }^{A}\right\}$.

particles were smaller. Therefore, in order to prepare smaller nanodrug by SAS process, the solution should be fed in relatively high speed.

With the increase of precipitation pressure, the average diameter decreased at first and then increased (Figure 2). This is mainly because the effect of precipitation pressure on the average diameter had a dual character. On one hand, the increase of precipitation pressure can lead to the increase of flow speed of supercritical carbon dioxide, and consequently growing time of crystal nuclei decreased. On the other hand, with the increase of precipitation pressure, the density of supercritical carbon dioxide increased, which led to concentrated precipitation of cefquinome from solution. In this case, crystal nuclei grew rapidly and agglomeration was more prone to appear.

From Figure 2, we can also find that when precipitation temperature increased, the average diameter became greater at first and then decreased. The reason was that the effect of precipitation temperature on the average diameter was multifaceted. The change of precipitation temperature can lead to the alterations of solubility of cefquinome, the solubility of solvent in supercritical carbon dioxide, the agglomeration, and other factors. Therefore, the selection of precipitation temperature depended on several factors.

3.3. Appearance Analysis of Processed Cefquinome Particles. SEM micrograph of the original cefquinome particles was shown in Figure 3. The appearance of these particles showed irregular blocks. The particle diameters ranged from 0.2 microns to 3 microns. SEM micrograph of processed cefquinome particles was shown in Figure 4. It can be seen that these particles were flaky. These particle diameters ranged from 0.1 microns to 1.2 microns. These particles aggregated together and formed larger particles. When these

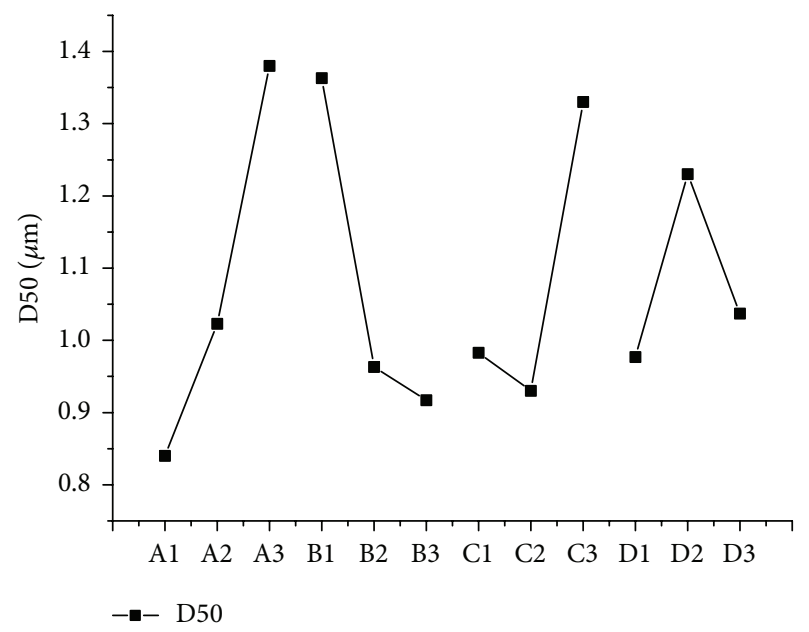

FIGURE 2: The effect of each factor on the average diameter of cefquinome particles. A: concentration of solution $(\mathrm{mg} / \mathrm{mL})$; B: solution feeding speed $(\mathrm{mL} / \mathrm{min})$; C: precipitation pressure (Mpa); D: precipitation temperature $\left({ }^{\circ} \mathrm{C}\right)$.

aggregated particles were dispersed in superpure water by supersonic wave, they could separate, which had been verified by laser particle size analyzer. These operations would occur in pharmaceutical industry. Therefore, the agglomeration cannot influence the efficacy of cefquinome nanoparticles in practical application.

\section{Conclusions}

Nanoparticles of cefquinome were prepared for the first time by using SAS process. By orthogonal experiments, it was confirmed that the concentration of solution was the primary 


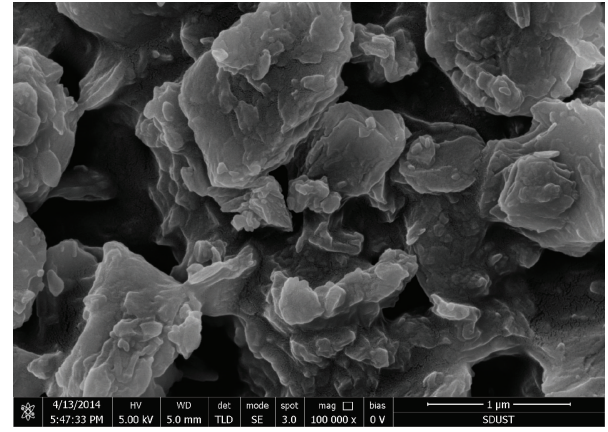

FIGURE 3: SEM micrograph of unprocessed cefquinome particles.

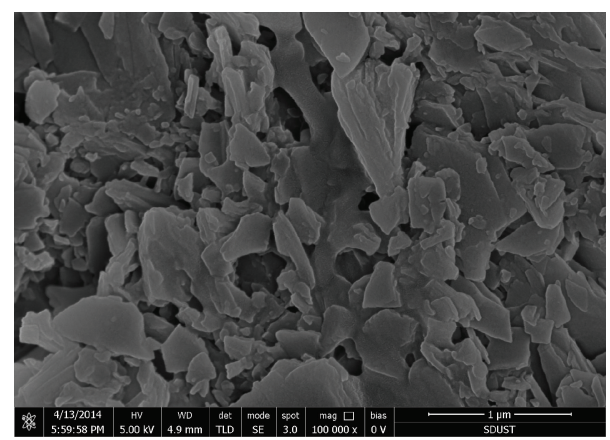

FIGURE 4: SEM micrograph of processed cefquinome particles.

factor in this process, followed by feeding speed of solution, precipitation pressure, and precipitation temperature. Meanwhile, the optimal operating conditions of preparing nanoparticles of cefquinome by supercritical antisolvent process were that solution concentration was $100 \mathrm{mg} / \mathrm{mL}$, solution flow speed was $1.5 \mathrm{~mL} / \mathrm{min}$, operating pressure was $13 \mathrm{Mpa}$, and operating temperature was $33^{\circ} \mathrm{C}$. Confirmatory experiment was conducted under this condition. The data showed that the appearance of particles was flakes and the average diameter of particles processed was 0.71 microns. Moreover, influence law of individual factor on particle size was investigated by univariate analysis. Results showed that the average diameter increased with increase of concentration of the solution but decreased with increase of feeding speed of the solution. The effect of both precipitation pressure and precipitation temperature on the average diameter was multifaceted. When these two factors increased, the average diameter could show extreme point. The process of preparing cefquinome nanoparticles by SAS method holds important implications for the efficacy enhancement of cefquinome and the development of pharmaceutical processes.

\section{Conflict of Interests}

The authors declare that there is no conflict of interests regarding the publication of this paper.

\section{References}

[1] V. Dinakaran, V. K. Dumka, B. Ranjan, R. Balaje, and P. K. Sidhu, "Pharmacokinetics following intravenous administration and pharmacodynamics of cefquinome in buffalo calves," Tropical Animal Health and Production, vol. 45, no. 7, pp. 1509-1512, 2013.

[2] A. M. Ehinger, H. Schmidt, and M. Kietzmann, "Tissue distribution of cefquinome after intramammary and 'systemic' administration in the isolated perfused bovine udder," Veterinary Journal, vol. 172, no. 1, pp. 147-153, 2006.

[3] J. M. Swinkels, T. J. G. M. Lam, M. J. Green, and A. J. Bradley, "Effect of extended cefquinome treatment on clinical persistence or recurrence of environmental clinical mastitis," Veterinary Journal, vol. 197, no. 3, pp. 682-687, 2013.

[4] K. G. Hopper, H. Leclair, and B. R. McCord, "A novel method for analysis of explosives residue by simultaneous detection of anions and cations via capillary zone electrophoresis," Talanta, vol. 67, no. 2, pp. 304-312, 2005.

[5] X. Zhao, X. Chen, Y. Zu, R. Jiang, and D. Zhao, "Recrystallization and micronization of taxol using the supercritical antisolvent (SAS) process," Industrial \& Engineering Chemistry Research, vol. 51, no. 28, pp. 9591-9597, 2012.

[6] M. Trotta, M. Gallarate, F. Pattarino, and S. Morel, "Emulsions containing partially water-miscible solvents for the preparation of drug nanosuspensions," Journal of Controlled Release, vol. 76, no. 1-2, pp. 119-128, 2001.

[7] J.-F. Chen, J.-Y. Zhang, Z.-G. Shen, J. Zhong, and J. Yun, "Preparation and characterization of amorphous cefuroxime axetil drug nanoparticles with novel technology: high-gravity antisolvent precipitation," Industrial \& Engineering Chemistry Research, vol. 45, no. 25, pp. 8723-8727, 2006.

[8] P. Sheth, H. Sandhu, D. Singhal, W. Malick, N. Shah, and M. S. Kislalioglu, "Nanoparticles in the pharmaceutical industry and the use of supercritical fluid technologies for nanoparticle production," Current Drug Delivery, vol. 9, no. 3, pp. 269-284, 2012.

[9] Y. Jiang, W. Sun, and W. Wang, "Recrystallization and micronization of 10-hydroxycamptothecin by supercritical antisolvent process," Industrial \& Engineering Chemistry Research, vol. 51, no. 6, pp. 2596-2602, 2012.

[10] E. Haimer, M. Wendland, A. Potthast, T. Rosenau, and F. Liebner, "Precipitation of hemicelluloses from DMSO/water mixtures using carbon dioxide as an antisolvent," Journal of Nanomaterials, vol. 2008, Article ID 826974, 5 pages, 2008.

[11] W. Z. He, Q. L. Suo, Z. H. Jiang, A. Shan, and H. L. Hong, "Precipitation of ephedrine by SEDS process using a specially designed prefilming atomizer," The Journal of Supercritical Fluids, vol. 31, no. 1, pp. 101-110, 2004.

[12] Y. Zhang, X. H. Zhao, W. G. Li, Y. Zu, Y. Li, and K. Wang, "Characterization, and dissolution rate in vitro evaluation of total Panax notoginsenoside nanoparticles, typical multicomponent extracts from traditional Chinese medicine, using supercritical antisolvent process," Journal of Nanomaterials, vol. 2015, Article ID 439540, 12 pages, 2015.

[13] E. Reverchon and G. Della Porta, "Production of antibiotic micro- and nano-particles by supercritical antisolvent precipitation," Powder Technology, vol. 106, no. 1-2, pp. 23-29, 1999.

[14] M.-S. Kim, S. Lee, J.-S. Park, J.-S. Woo, and S.-J. Hwang, "Micronization of cilostazol using supercritical antisolvent (SAS) process: effect of process parameters," Powder Technology, vol. 177, no. 2, pp. 64-70, 2007. 
[15] W. F. Li, G. J. Liu, L. X. Li, J. Wu, Y. Lü, and Y. Jiang, "Effect of process parameters on co-precipitation of paclitaxel and poly $(\mathrm{L}-$ lactic acid) by supercritical antisolvent process," Chinese Journal of Chemical Engineering, vol. 20, no. 4, pp. 803-813, 2012.

[16] M. Mukhopadhyay and S. V. Dalvi, "Mass and heat transfer analysis of SAS: effects of thermodynamic states and flow rates on droplet size," The Journal of Supercritical Fluids, vol. 30, no. 3, pp. 333-348, 2004.

[17] M. Rossmann, A. Braeuer, S. Dowy, T. G. Gallinger, A. Leipertz, and E. Schluecker, "Solute solubility as criterion for the appearance of amorphous particle precipitation or crystallization in the supercritical antisolvent (SAS) process," The Journal of Supercritical Fluids, vol. 66, no. 6, pp. 350-358, 2012.

[18] V. Patomchaiviwat, O. Paeratakul, and P. Kulvanich, "Formation of inhalable rifampicin-poly (L-lactide) microparticles by supercritical anti-solvent process," AAPS PharmSciTech, vol. 9, no. 4, pp. 1119-1129, 2008. 



Submit your manuscripts at http://www.hindawi.com
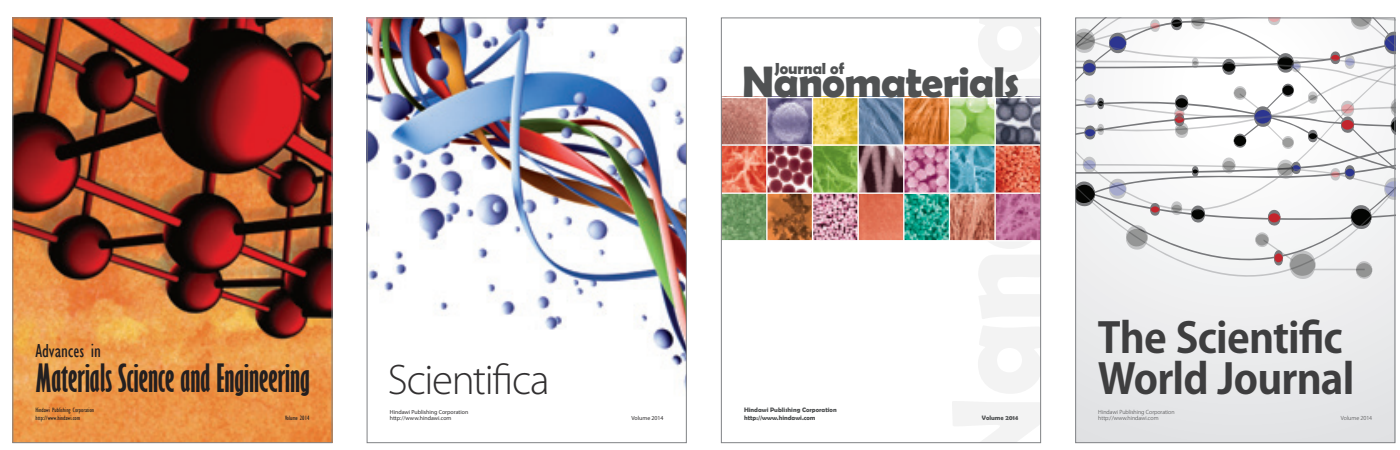

\section{The Scientific World Journal}
